\title{
BMJ Open Effect of female sex work-targeted community-based interventions along the HIV treatment cascade in sub- Saharan Africa: a systematic review protocol
}

\author{
Lydia Atuhaire (D) , ${ }^{1}$ Olatunji Adetokunboh, ${ }^{1,2,3}$ Constance Shumba, ${ }^{4,5}$ \\ Peter S Nyasulu (id) ${ }^{1,6}$
}

To cite: Atuhaire $L$, Adetokunboh 0, Shumba C, et al. Effect of female sex work-targeted communitybased interventions along the HIV treatment cascade in subSaharan Africa: a systematic review protocol. BMJ Open 2020;10:e039495. doi:10.1136/ bmjopen-2020-039495

- Prepublication history and supplemental material for this paper is available online. To view these files, please visit the journal online (http://dx.doi. org $/ 10.1136 /$ bmjopen-2020039495).

Received 16 April 2020 Revised 28 September 2020 Accepted 30 September 2020

Check for updates

(C) Author(s) (or their employer(s)) 2020. Re-use permitted under CC BY-NC. No commercial re-use. See rights and permissions. Published by BMJ.

For numbered affiliations see end of article.

Correspondence to

Lydia Atuhaire;

latuhaire@gmail.com

\section{ABSTRACT}

Introduction Female sex workers (FSWs) are a known high-risk group that are at increased risk of HIV transmission due to exposure to multiple sexual partners and inability to negotiate safe sex attributed to challenging economic circumstances. Previous systematic reviews have examined the effectiveness of HIV interventions prioritising FSWs and have shown that targeted interventions improve access to HIV prevention and treatment services. Interventions that increase FSWs' uptake of services are well documented; however, evidence on specific interventions aimed at improving FSWs' continuity in HIV care along the treatment cascade is lacking. This systematic review aims to document the performance of community-based interventions along the HIV treatment cascade.

Methods and analysis We will use a sensitive search strategy for electronic bibliographic databases, bibliographies of included articles and grey literature sources. In addition, the Joint United Nations Programme on HIV/AIDS and the WHO websites, peer-reviewed conference papers and grey literature sources will be searched for additional reports of sex work programmes. We will include randomised controlled trials, crosssectional surveys and cohort interventions where community-based HIV services were provided to FSWs and measure the performance of the HIV intervention on one or more cascade stages. We will conduct a systematic review of studies published from 2004 to present within the subSaharan Africa region. We will report quantitative study outcomes of HIV testing and diagnosis, linkage to care, initiation on antiretroviral therapy and viral suppression. We will analyse the data using the random-effects metaanalysis method, and funnel plots will be used to assess the publication bias.

Ethics and dissemination This systematic review will not require ethical approval; we will publish data from manuscripts. The results of this study will be disseminated in peer-reviewed journals and conference presentations.

PROSPERO registration number CRD42020157623.
Strengths and limitations of this study

- This protocol minimises duplication and allows transparency in the methods and processes used

- This protocol reduces possible biases and will enable peer review, hence a quality manuscript.

- This protocol will include only studies published in English language, and articles published in other languages may be missed.

- A limitation of this protocol is the scarcity of intervention studies on the effectiveness of communitybased interventions for female sex workers.

- The inadequate methodological quality on community-based interventions may be the limitation of this systematic review.

\section{INTRODUCTION}

Female sex workers (FSWs) are extremely affected with HIV compared with the general population. ${ }^{12}$ As a result, there is substantial interest in FSWs in the context of HIV due to their severe vulnerability and high susceptibility to acquiring HIV-known to be 21 times higher than other women of reproductive age. Furthermore, FSWs have a higher likelihood of transmitting HIV to their sexual partners due to having regular unsafe sex with multiple sexual partners attributed to challenging economic circumstances. ${ }^{3}$ FSWs are at an increased risk of HIV infection due to their inability to negotiate safe sex with sexual partners, high prevalence of sexually transmitted infections (STIs) and susceptibility to sexual violence. ${ }^{4}$ In addition, punitive environments such as violence, criminalisation, stigma and discrimination, and social and legal obstacles have been shown to limit access to services for HIV prevention, care and treatment for FSWs. ${ }^{67}$ 
Due to their susceptibility to HIV infection, various prevention efforts are focused on FSWs. ${ }^{8}$ Targeted interventions such as community-based HIV responses have been encouraged as models of care for FSWs. In 2018, the WHO developed a decision framework on differentiated antiretroviral therapy (ART) services, which encompasses HIV community responses for high-risk groups inclusive of FSWs. ${ }^{2}$ The framework provides guidance on implementation approaches that address the inequities in access by increasing acceptability, quality and coverage of health services by FSWs. ${ }^{9}$

Previous systematic reviews have assessed HIV behavioural interventions and sexual and reproductive health services for FSWs. ${ }^{10-13}$ Furthermore, other systematic reviews have assessed HIV prevalence ${ }^{14} 15$ and FSWs' HIV care cascade, access to HIV testing services, and HIV and treatment experiences. ${ }^{16-19}$ A systematic review that assessed ART uptake, attrition, adherence and outcomes among FSWs living with HIV $^{16}$ suggested that there is a concerning lack of published data for treatment response for FSWs. Nonetheless, the review data showed that FSWs can achieve the same treatment response and successful continuum of care compared with women in the general population. Another systematic review that examined HIV care experiences and determinants along the HIV treatment cascade among FSWs living with HIV in subSaharan Africa (SSA) showed that although ART has expanded, FSWs in SSA have suboptimal HIV care and treatment engagement outcomes. ${ }^{17}$ Other systematic reviews focused on HIV diagnosis and examined determinants of HIV counselling access and uptake among FSWs. ${ }^{18} 19$ These previous studies did not systematically assess specific interventions that impact FSWs' continuum of care outcomes, but rather presented collective factors that affect treatment outcomes across the cascade. Thus, they failed to identify and evaluate the uniqueness of specific interventions that may affect FSWs' continuation in HIV care such as community-based interventions.

Community-based HIV interventions for FSWs were assessed in two previous studies. The first one examined the effectiveness, challenges and considerations for implementation and scale-up among FSWs,${ }^{20}$ whereas the second one reviewed community empowerment interventions for HIV prevention among FSWs. ${ }^{21}$ According to the two studies, community-based empowerment approaches to addressing HIV among FSWs lead to reduction in HIV and STI transmission, increased access to condoms and result in general improvements in HIV outcomes. However, none of these studies systematically presented measurable outcomes attributed to FSWs' targeted community-based HIV interventions along the HIV treatment cascade.

The proposed systematic review will therefore aim to provide evidence on the effectiveness of communitybased interventions that provided HIV services to FSWs at community level by documenting an increase or decrease in access to HIV services along the treatment cascade. We will describe the proportions of those who tested for
HIV, got diagnosed and linked to care, were on ART and achieved viral suppression.

\section{METHODS AND ANALYSIS \\ Objectives}

The specific objective of this systematic review is to summarise data on the performance of prevention, care and treatment outcomes across the HIV treatment cascade attributed to targeted community-based interventions for FSWs in SSA.

\section{Criteria for considering studies for review}

We will include randomised controlled trials, crosssectional surveys and cohort interventions. We will also review unpublished quantitative data from reports and policy documents. Those that are published in English language will be included for review.

\section{Study participants}

The study participants are FSWs regardless of age. Studies reporting on community-based HIV services provided for FSWs in addition to other groups will also be included as long as data are disaggregated and reported by sex work status.

\section{Time and place}

This review will focus on studies conducted and published from 2004 to present within the SSA region. This will provide sufficient data to help understand challenges within the catchment area subscribed by Uganda, as well as the time span within which ART has been scaled up through widespread roll-out of comprehensive HIV programmes in SSA. ${ }^{22}$

\section{Intervention}

This review will target community-based interventions that provided targeted HIV services to FSWs. Targeted HIV services provided to FSWs in a clinic or drop-in centre setting in hotspot are also regarded as communitybased interventions.

\section{Outcomes}

Studies to be included must have measured and reported the performance of the community-based HIV intervention of focus after it was implemented and must have reported on one or more treatment cascade stages. In this review, the cascade stages include HIV testing, HIV diagnosis, linkage to care, on ART and viral suppression. Specifically, the information will be synthesised and will single out measurement of outcomes as follows:

- HIV testing: Reported increase in the number of FSWs who accessed HIV testing in a community-based HIV testing programme.

- HIV diagnosis: The proportion of FSWs diagnosed with HIV among those tested.

- Linked to care: The proportion of participants who got diagnosed versus the proportion of those who 
were linked to HIV care, defined as the completion of a first medical clinic visit after HIV diagnosis.

- On ART: The proportion of participants who are taking ART, among those who tested HIV-positive.

- Viral suppression: The proportion of participants living with diagnosed HIV who have attained viral suppression.

\section{Definition of FSWs' community-based service delivery}

For the purpose of this systematic review, the terms 'community-based service delivery' and 'communitybased HIV interventions' will be used interchangeably. In this review, these terms simply mean HIV interventions or services that are provided to FSWs outside of the formal public or private health facilities with the aim of improving health outcomes. The communitybased services to consider will have been implemented in various ways such as (1) those that provided supportive services to clinic-based care in the community setting such as linking the clients to the health system for HIV care, (2) routine outreach services, (3) targeted FSW clinics based in hotspots and (4) community services that were implemented on their own (stand-alone) without a link to the formal public or private health facilities.

\section{Definition of FSW}

In this review, the definition of an FSW is a woman who self-identified as a sex worker, engaged in transactional sex or exchanged sex for other gifts and commodities in the last 1 year.

\section{Exclusion criteria}

We will exclude qualitative studies, reviews, case studies, ecological studies, editorials and commentaries. Studies that did not measure or report the effect of communitybased HIV interventions on any of the cascade stage will also be excluded. We will exclude general discussion papers on community interventions and cascade stages that do not present data on impacts. Studies without a clear methodology on how community-based interventions were implemented and those not published in English will also be excluded.

\section{Search strategy}

We will use a sensitive search strategy for electronic bibliographic databases, bibliographies of included articles and grey literature sources. We will develop a comprehensive set of search terms subjectively and iteratively, checking MEDLINE (PubMed) to identify controlled vocabulary of Medical Subject headings (MeSH) terms related to our topic, and will also identify keywords based on our knowledge of the field. MEDLINE search terms will be adapted for other electronic databases to conform to their search functions. We will search the following electronic databases:

- MEDLINE (PubMed).

- CINAHL (EBSCO Host). Cumulative Index of Nursing and Allied Health Literature
- Science Citation Index Expanded and Social Sciences Citation Index, both from Web of Science.

- Scopus (Elsevier).

- Cochrane Library, including the Cochrane Central Register of Controlled Trials.

We will search the following websites: the Ministry of Health websites for countries with high population-level HIV prevalence, the Joint United Nations Programme on HIV/AIDS (UNAIDS) and WHO websites for additional reports of sex work programmes. We will also search for recent conference proceedings from the following conferences: International Conference on HIV/AIDS, International AIDS Society and International Conference on AIDS and STIs in Africa.

In addition to the electronic search, we will search the grey literature to identify any relevant unpublished reports. We will check the reference lists of potentially relevant articles for additional citations and will use the 'related citations' search key in PubMed to identify similar papers. The potential search terms will include HIV infections or HIV, treatment cascade or HIV continuum of care, community health services or community delivery services, sex workers or FSWs, and Africa South of the Sahara or sub-Saharan Africa. A detailed description of the extraction form is provided as online supplemental file 1. Search results will be managed using specialised bibliographic software (EndNote). We will prepare a detailed 'search diary' with details of the databases searched, keywords used and number of search results.

Data extraction, management and selection of eligible studies The initial screening of titles and abstracts will be undertaken against inclusion and exclusion criteria by two reviewers (LA, OA). All retrieved full-text studies will be assessed for relevance by the two reviewers independently (LA, OA), before they are included for the actual systematic review. Any disagreements will be resolved by mutual consensus or consultation with a third reviewer (PN or CS). Data will be extracted systematically using a standardised tool that will be developed based on the format in the Cochrane Handbook for Systematic Reviews of Interventions. ${ }^{23}$ The developed tool will be piloted by two reviewers (LA, OA) independently on a random sample of five ${ }^{5}$ articles, and discrepancies in extracted data will be resolved by consensus and the tool revised accordingly.

In the event that data on key variables are missing, or we require any other clarification about the study, we will contact the study authors to furnish us with the required information. A description of missing data for each study will be documented and possible implications of missing data discussed.

\section{Categorising studies}

During the data extraction, studies will be categorised by the type of community-based intervention and outcome. If the data on categorisation are not found in the studies, the authors of such articles will be contacted for further information. For studies that evaluated 'integrated 
community-based' interventions with no disaggregated data per intervention, the data will be extracted in that format and the results presented as they are, if they had reported on outcomes of one of the cascade stages.

\section{Data analysis}

The data will be analysed using random-effects metaanalysis where applicable. First, we will synthesise the main characteristics of included studies and use summary statistics to describe study characteristics such as mean (SD), median and frequencies. For each step of the cascade together with the extracted community HIV service delivery interventions, we will calculate proportions with exact binomial 95\% CIs and present these in forest plots.

We will calculate the homogeneity of the results by means of the $\chi^{2}$ test and use the $\mathrm{I}^{2}$ to describe the percentage variation across included studies. ${ }^{23}$ We do not anticipate more variation than would be expected because the participants (FSWs), the intervention (community-based service delivery interventions) and outcomes (performance on cascade stages) are all expected to be interlinked. However, due to variations in the study design and methodology, we expect some level of between-study heterogeneity, and the focus of the subsequent analysis will therefore be on the identification and exploration of sources of heterogeneity. We will explore substantial heterogeneity $\left(\mathrm{I}^{2}>50 \%\right)$ by subgroup analysis. All analyses will be done in R V.3.6.1 (R Foundation, Vienna, Austria).

In this proposed review, we expect to get adequate data to perform a meta-analysis because of anticipated homogeneity in relation to participants, interventions and outcome measures. However, if we find that the identified studies are not homogeneous enough in terms of methodology and outcome measures, meta-analyses of the data will not be performed.

\section{Risk of bias in included studies}

We will assess risk of bias both at study and at outcome level for the included non-randomised studies of interventions using the risk of bias in non randomised studies of interventions-ROBINS-I risk of bias tool..$^{24}$ Risk of bias of randomised studies will be assessed using the Cochrane 'risk of bias' tool ${ }^{25}$; risk of bias of cross-sectional and cohort studies will be assessed and rated for quality using the adapted Newcastle Ottawa Scale. ${ }^{26}$ Publication bias will be assessed by visually inspecting funnel plot asymmetry and by including study size in the logistic model. The quality assessment will be cross-checked, and any disagreement will be resolved by consensus within the review team.

The strength of the body of evidence will be assessed using the Grading of Recommendations Assessment Development and Evaluation (GRADE) approach. ${ }^{27}$ The GRADE certainty of evidence categorised as high, moderate, low and very low will be determined based on an assessment of evidence for risk of bias, publication bias and indirectness.

\section{Patient and public involvement}

There were no patients and public involvement in the design of this protocol; however, the results from the systematic review will be disseminated to FSW organisations that we are already working with for other studyrelated objectives. Furthermore, the results will be disseminated through conference presentations to the Ministry of Health Key Population Technical Working Group, which will in turn release the findings to additional FSW organisations and other stakeholders.

\section{Ethics and dissemination}

This systematic review will not require ethical approval; we will publish data from manuscripts. The results of this study will be disseminated in peer-reviewed journals and conference presentations, seminars and symposia.

\section{DISCUSSION}

Targeted interventions focused on FSWs' specific challenges related to access to and retention in HIV care are required. ${ }^{2}$ Understanding which particular interventions optimally affect access to care, as well as retention in care among FSWs living with HIV across the HIV treatment cascade, is important as programmes strive to improve the treatment outcomes. However, studies have mainly focused on combined effects of interventions on access to and continuity in HIV care, without distinguishing those that optimise continued engagement in care by FSWs. ${ }^{16} 17$ As a result, the benefits of determining where specific interventions may be more effective along the care cascade are lost. This also undermines the opportunity to increase allocative efficiency for interventions.

In this review, we will be able to examine the extent to which FSWs' targeted community-based interventions are contributing to FSWs' access to HIV testing and care, retention in care and achievement of viral suppression. Owing to the strong emphasis on scale-up of community responses with the greatest impact, ${ }^{28}$ the results from this review will provide insight on the organisation of data collection systems used in community HIV interventions, how measurements have been carried out and the challenges that still need to be addressed.

Furthermore, the evidence for the effectiveness of community-based interventions that will be provided by this systematic review will contribute to the call by UNAIDS and other multilateral partners to all HIV response stakeholders, to refine methodologies to rigorously assess and determine which approaches work better and to create impact to allocate resources efficiently. The evidence gathered from this review will be key to guide and inform policy as well as support programming of community-based HIV interventions that optimally improve FSWs' access to and continuity in HIV care for improved HIV outcomes. This systematic review will, for the first time, provide measurable evidence on how different community-based service delivery interventions 
directly affect specific stages within the continuum of care for FSWs.

\section{Author affiliations}

${ }^{1}$ Division of Epidemiology \& Biostatistics, Faculty of Medicine and Health Sciences, Stellenbosch University, Cape Town, South Africa

${ }^{2}$ Cochrane South Africa, South African Medical Research Council, Cape Town, South Africa

${ }^{3}$ DSI-NRF Centre of Excellence in Epidemiological Modelling and Analysis (SACEMA), Stellenbosch University, Cape town, South Africa

${ }^{4}$ School of Nursing and Midwifery, Aga Khan University East Africa, Nairobi, Kenya ${ }^{5}$ Department of Population Health, Aga Khan University East Africa, Nairobi, Kenya ${ }^{6}$ School of Public Health, Faculty of Medicine and Health Sciences, University of the Witwatersrand, Johannesburg, South Africa

\section{Twitter Peter S Nyasulu @pnyasulu}

Acknowledgements We thank Dr Vittoria Lutje, Mrs Ingrid van der Westhuizen and Ms Pamela Nyokwana for their support in the development of the search strategies.

Contributors LA conceptualised the study, drafted the manuscript and designed the search strategy and study selection process. PSN, CS and OA contributed to critically reviewing conceptualisation and design of the study protocol. All authors read, provided feedback and approved the final version of the protocol. PSN is the guarantor.

Funding The authors have not declared a specific grant for this research from any funding agency in the public, commercial or not-for-profit sectors.

Competing interests None declared.

Patient consent for publication Not required.

Provenance and peer review Not commissioned; externally peer reviewed.

Supplemental material This content has been supplied by the author(s). It has not been vetted by BMJ Publishing Group Limited (BMJ) and may not have been peer-reviewed. Any opinions or recommendations discussed are solely those of the author(s) and are not endorsed by BMJ. BMJ disclaims all liability and responsibility arising from any reliance placed on the content. Where the content includes any translated material, BMJ does not warrant the accuracy and reliability of the translations (including but not limited to local regulations, clinical guidelines, terminology, drug names and drug dosages), and is not responsible for any error and/or omissions arising from translation and adaptation or otherwise.

Open access This is an open access article distributed in accordance with the Creative Commons Attribution Non Commercial (CC BY-NC 4.0) license, which permits others to distribute, remix, adapt, build upon this work non-commercially, and license their derivative works on different terms, provided the original work is properly cited, appropriate credit is given, any changes made indicated, and the use is non-commercial. See: http://creativecommons.org/licenses/by-nc/4.0/.

\section{ORCID iDs}

Lydia Atuhaire http://orcid.org/0000-0002-6683-0862

Peter S Nyasulu http://orcid.org/0000-0003-2757-0663

\section{REFERENCES}

1 UNAIDS.. UNAIDS data. In: Joint United nations programme on HIVI AIDS. Geneva, Switzerland, 2019.

2 WHO. Differentiated service delivery for HIV: a decision framework for differentiated antiretroviral therapy delivery for key populations. Geneva: World Health Organization, 2018.

3 Ritchie H. Causes of death. Our World In Data org, 2018.

4 Prüss-Ustün A, Wolf $\mathrm{J}$, Driscoll T, et al. HIV due to female sex work: regional and global estimates. PLoS One 2013;8:e63476.

5 Macdonald V, Verster A, Baggaley R. A call for differentiated approaches to delivering HIV services to key populations. J Int AIDS Soc 2017;20:21658.
6 Matovu JKB, Ssebadduka NB. Knowledge, attitudes \& barriers to condom use among female sex workers and truck drivers in Uganda: a mixed-methods study. Afr Health Sci 2013;13:1027-33.

7 Decker MR, Wirtz AL, Moguilnyi V, et al. Female sex workers in three cities in Russia: HIV prevalence, risk factors and experience with targeted HIV prevention. AIDS Behav 2014;18:562-72.

8 Aral SO, Blanchard JF. Phase specific approaches to the epidemiology and prevention of sexually transmitted diseases. Sex Transm Infect 2002;78:i1-2.

9 Macdonald V, Verster A, Baggaley R. A call for differentiated approaches to delivering HIV services to key populations. J Int AIDS Soc 2017;20:21658

10 Ferguson A, Shannon K, Butler J, et al. A comprehensive review of HIV/STI prevention and sexual and reproductive health services among sex workers in Conflict-Affected settings: call for an evidence- and rights-based approach in the humanitarian response. Confl Health 2017;11:25.

11 Dhana A, Luchters S, Moore L, et al. Systematic review of facilitybased sexual and reproductive health services for female sex workers in Africa. Global Health 2014;10:46.

12 Chow EPF, Tung K, Tucker JD, et al. Behavioral interventions improve condom use and HIV testing uptake among female sex workers in China: a systematic review and meta-analysis. AIDS Patient Care STDS 2015;29:454-60.

13 Chersich MF, Luchters S, Ntaganira I, et al. Priority interventions to reduce HIV transmission in sex work settings in sub-Saharan Africa and delivery of these services. $J$ Int AIDS Soc 2013;16:17980.

14 Platt L, Jolley E, Rhodes T, et al. Factors mediating HIV risk among female sex workers in Europe: a systematic review and ecological analysis. BMJ Open 2013;3:e002836.

15 Baral S, Beyrer C, Muessig K, et al. Burden of HIV among female sex workers in low-income and middle-income countries: a systematic review and meta-analysis. Lancet Infect Dis 2012;12:538-49.

16 Mountain E, Mishra S, Vickerman P, et al. Antiretroviral therapy uptake, attrition, adherence and outcomes among HIV-infected female sex workers: a systematic review and meta-analysis. PLoS One 2014;9:e105645.

17 Lancaster KE, Cernigliaro D, Zulliger R, et al. HIV care and treatment experiences among female sex workers living with HIV in subSaharan Africa: a systematic review. Afr J AIDS Res 2016;15:377-86.

18 Nnko S, Kuringe E, Nyato D, et al. Determinants of access to HIV testing and counselling services among female sex workers in subSaharan Africa: a systematic review. BMC Public Health 2019;19:15.

19 Tokar A, Broerse JEW, Blanchard J, et al. HIV testing and counseling among female sex workers: a systematic literature review. AIDS Behav 2018;22:2435-57.

20 Kerrigan D, Kennedy CE, Morgan-Thomas R, et al. A community empowerment approach to the HIV response among sex workers: effectiveness, challenges, and considerations for implementation and scale-up. Lancet 2015;385:172-85.

21 Kerrigan DL, Fonner VA, Stromdahl S, et al. Community empowerment among female sex workers is an effective HIV prevention intervention: a systematic review of the peer-reviewed evidence from low- and middle-income countries. AIDS Behav 2013;17:1926-40.

22 Egger M, Boulle A, Schechter M, et al. Antiretroviral therapy in resource-poor settings: scaling up inequalities? Int $J$ Epidemiol 2005;34:509-12.

23 Higgins J. Cochrane Handbook for systematic reviews of interventions. Version 5.1. 0. Cochrane Database Syst Rev, 2011.

24 Sterne JA, Hernán MA, Reeves BC, et al. ROBINS-l: a tool for assessing risk of bias in non-randomised studies of interventions. BMJ 2016;355:i4919.

25 Higgins JPT, Altman DG, Gotzsche PC, et al. The Cochrane collaboration's tool for assessing risk of bias in randomised trials. BMJ 2011;343:d5928.

26 Wells GA, Shea B, O'Connell D, et al. The Newcastle-Ottawa scale (NOS) for assessing the quality of non randomized studies in metaanalyses, 2011.

27 Schünemann HJ, Schünemann AHJ, Oxman AD, et al. Grading quality of evidence and strength of recommendations for diagnostic tests and strategies. BMJ 2008;336:1106-10.

28 Ortblad KF, Oldenburg CE. Tailoring combination HIV prevention for female sex workers. Lancet HIV 2018;5:e406-7. 